\title{
Understanding Structure-Property Relationships in Nanoparticle Electrocatalysts through Correlated Electron Microscopies
}

\author{
See Wee Chee ${ }^{1}$, Aram Yoon ${ }^{2}$, Fengli Yang ${ }^{1}$, Philipp Grosse ${ }^{1}$, Jeffery Poon ${ }^{1}$ and Beatriz Roldan \\ Cuenya $^{1}$ \\ ${ }^{1}$ Department of Interface Science, Fritz Haber Institute of the Max Planck Society, United States, \\ ${ }^{2}$ University of Illinois at Urbana-Champaign, United States
}

Renewable energy-powered electrocatalytic conversion of simple, environmentally-abundant molecules, such as $\mathrm{H}_{2} \mathrm{O}$ and $\mathrm{CO}_{2}$, into useful fuels and chemicals is an important strategy for moving our society's towards sustainable development [1], [2]. However, many of these approaches are not economically viable yet because of the limitations of current electrocatalysts. Nanoparticles are attractive candidates for electrocatalytic applications owing to their high surface area to volume ratios and unique structure-derived properties. However, obtaining catalysts that are optimized in terms of activity, selectivity and stability is also challenging due to our incomplete understanding of the structure of catalysts that exists under reaction conditions [3]. Although there have been significant advances in operando techniques that allow us to investigate the transformations that take place during reaction [4], most of these techniques are ensembleaveraging and thus, provide little insight into the local heterogeneities that can be found in nanoparticle catalysts.

Electrochemical liquid cell transmission electron microscopy (EC-TEM) has seen rapid growth in recent years [5] and is a promising approach for studying the dynamic behavior of nanoparticle electrocatalysts. Nevertheless, to rationalize the structure-property relationships in these materials, it is necessary that we extend the in situ observations across the length scales and develop methods that can measure the performance of the catalysts within the microfabricated electrochemical cells used for EC-TEM. In this presentation, I will describe the correlated electron microscopy approaches that we are exploring at the Fritz Haber Institute to address these challenges.

To obtain detailed insight into the behavior of electrocatalysts across different length scales, we use a combination of in situ transmission electron microscopy (TEM) and scanning electron microscopy (SEM) setups with similar electrochemical configurations (Figure 1). TEM provides the high-resolution image sequences that describe the behavior of catalysts under applied potential, whereas SEM provides comparable image sequences at lower spatial resolution but encompass a larger field-of-view with many catalyst nanoparticles. These holders also incorporate inlets for regular reference and counter electrodes to enable an accurate comparison with the results from benchtop electrochemical experiments. An additional ex situ TEM holder further allows us to perform detailed identical location characterization of the samples on the electrochemistry chips before and after reaction (Figure 1).

The potential of such an approach for illuminating the dynamics that are present under reaction conditions is illustrated with experiments looking at the behavior of model electrocatalysts for carbon dioxide reduction and oxygen evolution. Opportunities for using the larger chamber of the SEM for adding complementary characterization capabilities will also be discussed. 


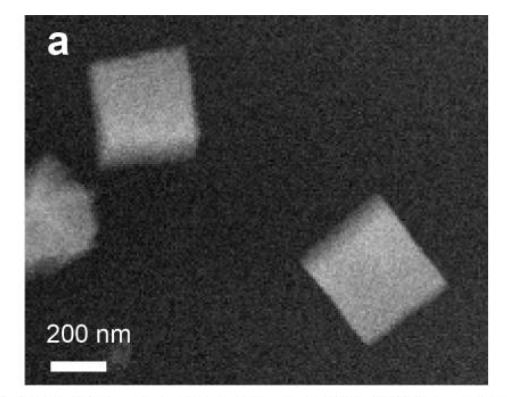

High Resolution Dynamic Behavior from Electrochemical TEM
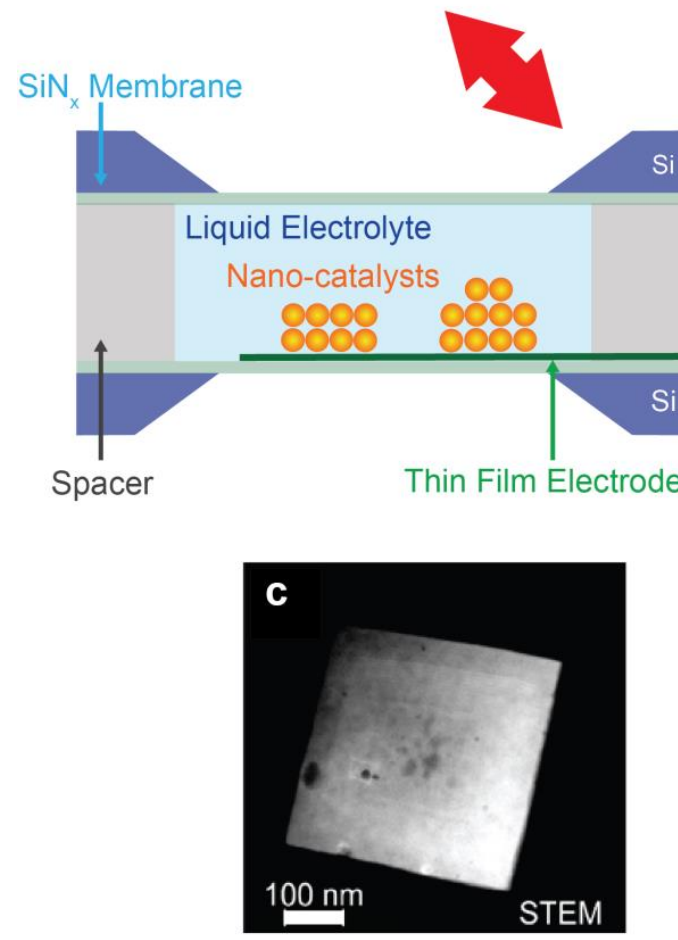

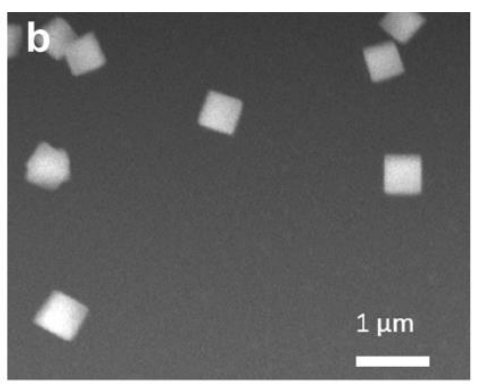

Particle Statistics and Complementary Analysis from Electrochemical SEM

\section{Transformations under}

Reaction Conditions

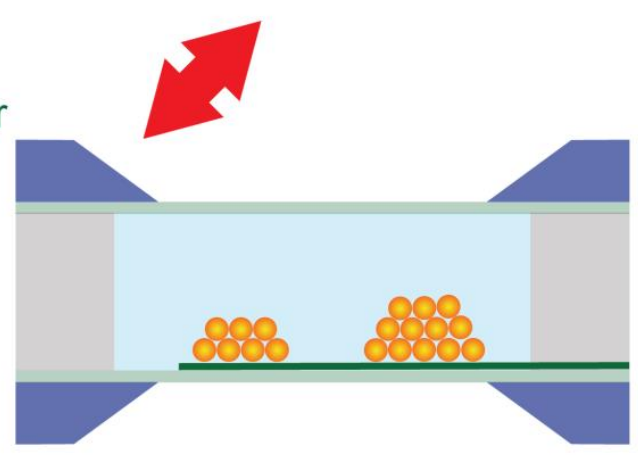

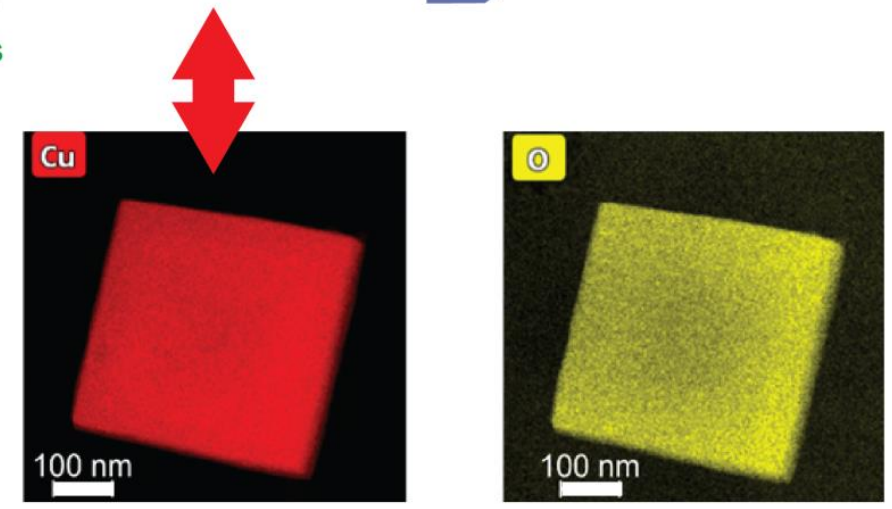

Ex Situ Identical Location TEM Investigations

Figure 1. Figure 1. Schematic describing the concept of using correlated electron microscopies to investigate the transformations of electrocatalysts under reaction conditions. The images depict STEM images and EDX maps of cubic $\mathrm{Cu} 2 \mathrm{O}$ model catalysts for the electrochemical reduction of carbon dioxide.

(b) and (c) are modified from [6] and [7] respectively.

\section{References}

[1] C.M. Friend and B. Xu, Acc. Chem. Res., 50 (2017) pp. 517-521

[2] Z.W. Seh et al., Science, 355 (2017) no. 6321

[3] H. Mistry et al., Nat. Rev. Mater., 1(2016), pp. 16009

[4] A. Bergmann and B. Roldan Cuenya, ACS Catal., 9 (2019), pp. 10020-10043

[5] F. M. Ross, Science, 350 (2015), no. aaa9886

[6] Yoon et al., Microsc. Microanal., 27 (2021), pp. 121 - 128

[7] Grosse et al., J. Phys. Chem. C, 124 (2020), pp. 26908-26915 